\title{
Balloon overtube-assisted placement of self-expanding metal stents
}

Occasionally, placement of a self-expanding metal stent (SEMS) into the distal stomach, colon, or small bowel may not be possible because of an angulated or torqued lumen [1]. This problem can be solved by either advancing the stent over stiffer wires or by through-the-scope placement. However, the large-diameter delivery system of esophageal SEMS impedes its application through the endoscopic channel. Here we present a novel balloon overtube-assisted technique for successful placement of SEMS in patients whose upper gastrointestinal tract has deranged, tortuous, and/or stenotic lumens. Our first case was a 61-year-old man with a massive gastric cancer with concealed perforation and a large necrotic cavity. He was a poor surgical candidate and the objective was to bridge the stomach to the duodenum. The second case was a 38-year-old man who was referred for a gastric-peritoneal fistula and stenosis of a sleeve gastrectomy carried out 4 months previously. In both patients it was impossible to advance the fully covered metal stent over previously placed hard (spring-tipped Savary) or soft (biliary) wires. Due to the tortuous anatomy, the delivery device developed kinking or could not follow the wires ( Fig. 1). Therefore, a balloon overtube (Fujifilm, Tokyo-Saitama, Japan) was placed over the scope and advanced across the perforation or sleeve, respectively ( Fig.2). We chose to use a balloon overtube for several reasons: it has a ring mark distally; it is relatively soft, permitting sliding across partially stenotic areas; and it has a balloon at the tip, which may allow inflation or anchoring. Because the overtube is larger than the gastroscope, an incision was made on its side to allow introducing the scope, as described previously [2]. After the overtube had been passed distally and a wire had been placed deeply into the upper jejunum, the scope was removed and the stent was placed over the wire ( Fig.3). The overtube was then carefully retrieved while the stent was being deployed ( $\bullet$ Fig. 4 ). This maneuver was essential to prevent stent deployment within the overtube. The stents were deployed satisfactorily in each case. Both patients improved markedly with this therapy.

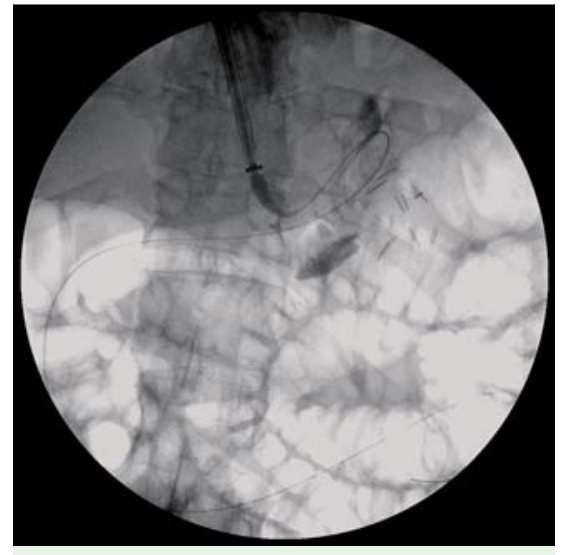

Fig. 1 Due to the tortuous anatomy in both patients, the delivery device kinked or could not follow the wires.

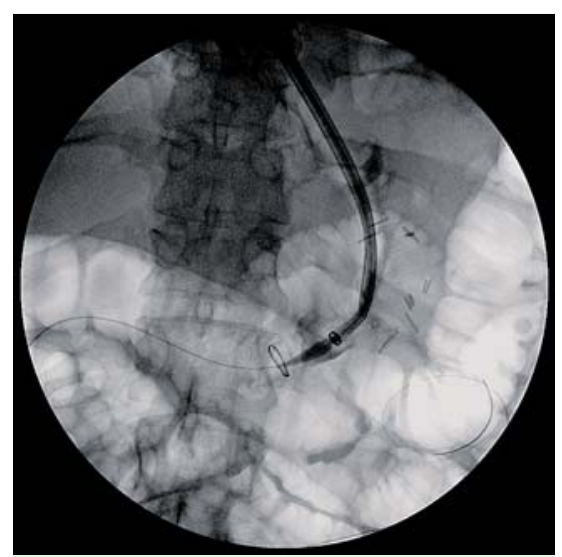

Fig. 3 After the overtube had been passed distally and a wire had been placed deeply into the upper jejunum the scope was removed and the stent was placed over the wire.

These cases clearly show that balloon overtubes can be used to deliver SEMS into the desired position, even in patients with severe anatomical deformities due to underlying malignant or benign pathologies. The overtube assumes two important functions for stent placement. First, it acts as a stabilizer and straightener of the deformed gastrointestinal tract. Second, the overtube serves as an "extralarge" working channel, which makes advancing the stent (or other devices) over the wire much easier. A key point to remember when using this technique is to pull the overtube proximally while the

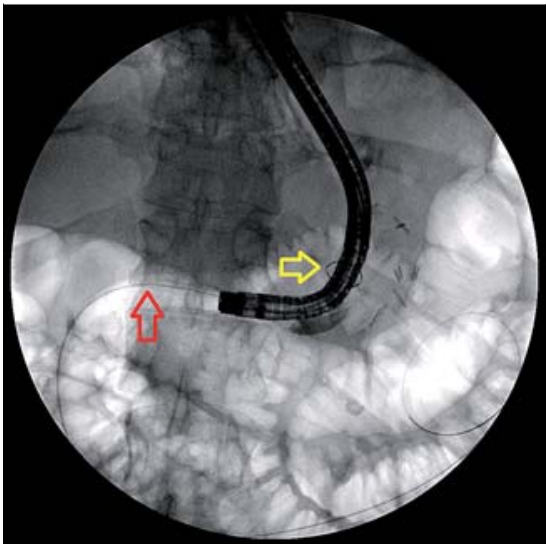

Fig.2 A balloon overtube was placed over the scope and advanced across the perforation or sleeve. The balloon overtube has a ring mark at its tip (yellow arrow). The wire is in the jejunum (red arrow).
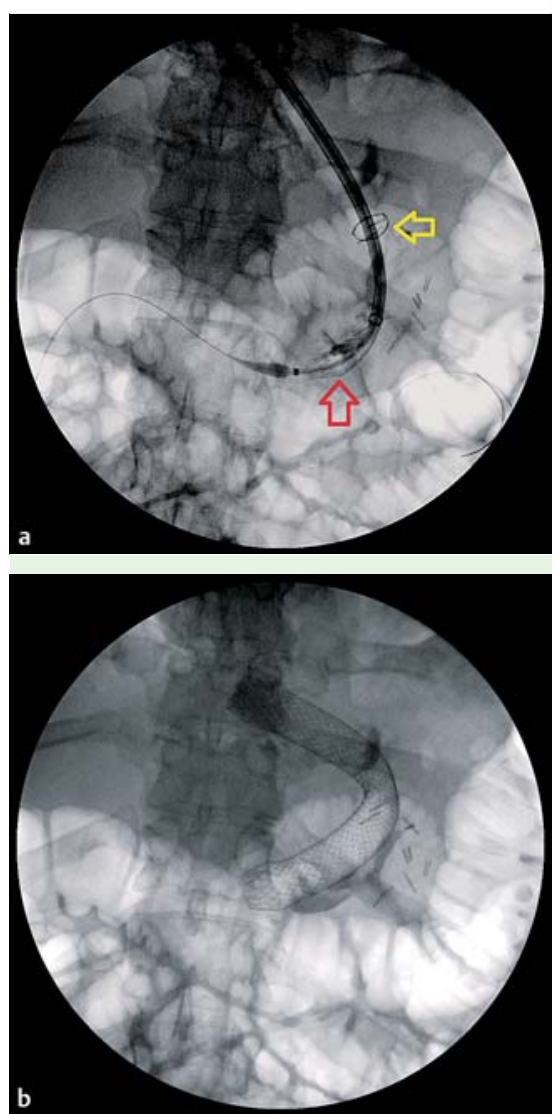

Fig.4 a The overtube was carefully retrieved (the yellow arrow is indicating the ring of the overtube) while the stent was being deployed (red arrow). This maneuver is essential to prevent stent deployment within the overtube. b The fully deployed metal stent. 
stent is being deployed distally, so as to avoid deployment of the stent within the overtube. In sum, this newly introduced overtube-technique for stent placement should be kept in mind when dealing with complex or distorted upper gastrointestinal anatomy.

Endoscopy_UCTN_Code_TTT_1AO_2AZ

\section{Competing interests: None}

\section{H. Neumann ${ }^{1,2}$, C. M. Wilcox ${ }^{1}$, K. Mönkemüller ${ }^{1}$}

${ }^{1}$ Basil Hirschowitz Endoscopic Center of Excellence, Division of Gastroenterology and Hepatology, University of Alabama at Birmingham, Birmingham, United States of America

2 Department of Medicine 1, Interdisciplinary Endoscopy, University of ErlangenNuremberg, Erlangen, Germany

\section{Acknowledgment}

Helmut Neumann is a recipient of the 2013 ASGE Cook Medical Don Wilson Award. This work was done during his awardee period at the Basil Hirschowitz Endoscopic Center of Excellence, University of Alabama, Birmingham, USA.

\section{References}

1 Baron $T H$. Expandable metal stents for the treatment of cancerous obstruction of the gastrointestinal tract. N Engl J Med 2001; 344: $1681-1687$

2 Fähndrich M, Sandmann M, Heike M. A facilitated method for endoscopic interventions at the bile duct after Roux-en-Y reconstruction using double balloon enteroscopy. $\mathrm{Z}$ Gastroenterol 2008; 46: 335-338

\section{Bibliography}

Dol http://dx.doi.org/

10.1055/s-0033-1344767

Endoscopy 2013; 45: E369-E370

(c) Georg Thieme Verlag KG

Stuttgart · New York

ISSN 0013-726X

\section{Corresponding author}

\section{K. Mönkemüller}

Division of Gastroenterology and Hepatology Basil Hirschowitz Endoscopic Center of Excellence Endoscopy Unit, JT 664

619 19th

Street S

Birmingham

AL 35249

USA

klaus1@uab.edu 